\title{
ZU ANTHOLOGIA LATINA ED. RIESE No.285 UND 285 a (DE CONVIVIIS BARBARIS).
}

Die herstellung und deutung der in dem bekannten epigramm ïberlieferten gotica eils .... scapiamatziaiadrincan ist trotz vielfacher versuche zur lösung der frage bis jetzt eine controverse geblieben.

Massmanns Hails sijais! skap jah matjan jah drigkan! = 'Sei gegrüisst (du eintretender)! (kellner), bringe (ihm) sowol zu essen als zu trinken!' (s. Zs. fda. 1، 379 ff.) oder Hails sijais! skap! jah matjam jah drigkam! = 'Sei gegrüsst (du eintretender)! (kellner) einen becher! lasst uns sowol essen als trinken!' könnte zur not dem sinne nach befriedigend erscheinen, wenn es auch so auffallen dürfte, dass der dichter worte als charakteristica des lärms der zechbrüder gewählt hätte, die nur beim eintritt eines neu angekommenen genossen, also verhältnismässig selten gehört wurden. $\mathrm{Zu}$ beanstanden sind dagegen in sprachlicher hinsicht: die ansetzung eines imperativs skap (statt skapei) und die (übrigens auch von den anderen commentatoren postulierte) annahme einer erst für das jüngere germanisch bezeugten verwendung dieses verbs für 'bringen', 'praebere' (man beachte got. gaskapjan $\approx \tau i \zeta \varepsilon \iota \nu$, ahd. scaffan, scepfen creare, haurire, as. slieppian haurire, ags. sceppan creare, constituere etc.), bez. die übersetzung von skap (= ahd. scaf; as. skap, ags. sceap, afries. scep dolium, tina, haustrum) durch 'becher'. Metrische bedenken erregt überdies ein hexameter

Inter | hails si|jais goti|cum skap ja|matjan (-am) ja $\mid$ drincan (-am)

in dem einerseits $r+h$ zweifache consonanz repräsentieren, andrerseits $p+j$ und $n(m)+j$ keine position bilden sollten, 
ferner das si- eines eventuell von dem Römer gehörten und reproducierten sijais als länge, aber der vocal von $j \bar{a}$ (für j $\bar{a} \bar{h}$ aus iaßたe, vgl. IF. 14, 83) entgegen der vom Römer gehörten quantität als kürze zu gelten hätte.

J. Grimm lässt bei seinem vorschlag (s, GDS. ${ }^{3}$ 318)

Inter hails gothicum skapjam atazja jah drigkam

das metrum ganz ausser acht, misst willkürlich, unter anziehung von ahd. azzasi utensilia, seinem atazja die bedeutung 'pocula' bei und übersieht das auffallende einer lat. darstellung von got. sibilans durch z. Aus skapjam atazja "paremus pocula' müsste ausserdem als consequenz folgen, dass die zechende gesellschaft es für nötig hielt, dem ankömmling mehrere becher zur verfügung zu stellen.

Auch das von Dietrich (Ueber die aussprache des gotischen s. 26) befürwortete

Inter | heils goti $\mid$ cum sca $|p \bar{\imath} j a|$ matja' ja|drincan (= 'schaffe sowol zu essen als zu trinken') wird dem metrischen gar wenig gerecht: ein $\bar{a}$ in scapp $\bar{\imath}$ zweierlei messuing von ja (das erste als $j \bar{a}$, das andere als $j a$ ) und elision des nasals von matjan vor $j$ (beachte im 2. vers des epigramms quis|quam dig|nos) wären schwerlich denkbar.1)

Dem von Luft (s. Zs. fda. anz. 23, $392 \mathrm{ff}$ ) in seiner lesart

Inter geils goticum scapjī (aus gekürzem scapi 'schaffe' $+\bar{\imath}$ 'dass') ja gamatjam ja drincam

unter berufung von westgerm. geil (gèl, zál) laetus, elatus und

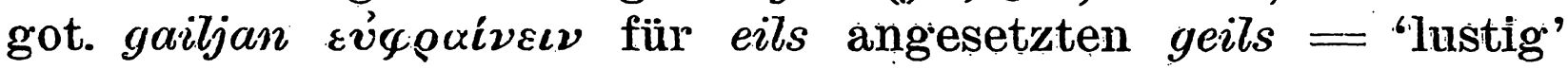
könnte man insofern einen vorzug gewähren, dass es der annahme von Inter | hails (heils) überhebt, deren bedenklichkeit auch durch Möllers bemerkung (Zs.fda. anz. 25, 104) 'das gotische anlautende $h$ war eben, im gegensatz zum lat. $h$, das ein nichts war, noch ein etwas, das ... als ein etwas vom dichter gefasst werden konnte, indem es mit dem $-r$ position bildet' nicht beseitigt wird: dass der römische dichter einem intensiveren got. hauchlaut $\mathrm{zu}$ liebe seine lat. prosodie verleugnet hätte, wäre ja kaum anzunehmen. Doch möchte man andrer-

i) Nach Zs. fda. anz. 6, 374 stinmt mit der Dietrich'schen fassung eine von Grabow in der Festgabe an Aug. Stinner veröffentlichte deu.tung des verses überein. Leider war es mir nicht möglich, dieser schrift selbst habhaft zu werden. 
seits diesem geils seines problematischen charakters wegen kein grosses vertrauen entgegenbringen und, was den schlussteil des verses betrifft, mit Möller (a.a.o.) bezweifeln, ob der forderung, dass die gotischen worte sich denselben metrischen regeln fügen, nach denen die lateinischen worte gesetzt sind, durch Lufts ansatz in der tat genüge geleistet sei: einem

\section{-j̄̄ ja ga|matjam ja|drincam}

haften eben die bereits oben gerügten metrischen fehler an. Dasselbe gilt, mit ausnahme der elision des nasals, für das nach L. ev. für die erwähnte lesart zu substituierende (und dann wol als äusserung des eintretenden $\mathrm{zu}$ fassenden) scapj $\bar{\imath}$ ja gamatja ja drinca (= 'schaffe, kellner, dass ich sowol esse als trinke'). Als eine annahme ad hoc hat übrigens noch das gekürzte scapi zu gelten.

Gegen Möllers vorschlag (s. Deutsche rundschau 32, 416, anm. und Zs. fda. anz. 25, 103 f.)

Inter | heils goti|cum scap|jam mat|jan ja|drincan (= 'lasst uns $\mathrm{zu}$ essen und trinken schaffen') ist ausser den zuvor erwähnten, heils und scapjam betreffenden einwänden auch die unstatthaftigkeit eines mit drei spondeen schliessenden hexameters geltend zu machen. Ausserdem wäre hier wol eher ein imperativ als ein adhortativ am platze.

Angesichts des erörterten dürfte also ein neuer erklärungsversuch gerechtfertigt sein, jedoch nur ein solcher, der dem sinne nach sowie sprachlich und metrisch befriedigen könnte, zugleich aber möglichst auf mehr oder weniger willkürliche änderungen der überlieferung verzichtet.

Dass dem dichter nicht das etwa beim eintritt eines neuen zechgenossen gehörte, sondern vielmehr einige während des gelages widerholt erschallenden worte als zur typischen bezeichnung des zechenlärms geeignet erscheinen mussten, liegt auf der hand. Als solche worte aber begreifen sich:

ein heils, das, wie bekannt, als bègrüssungsformel = 'salve', aber auch als ausruf beim zutrinken in schwang war [vgl. Grimms Gramm. 4, 298 sowie Dietrich a. a. o. und beachte den passus in Venantius Fortunatus' Praefatio ${ }^{1}$ ), worin der poet über sein auftreten vor germanischen hörern berichtet: ut inter

1) MG. Auct. antiqu. IV, pars prior, s. 2. 
illos egomet non musicus poeta, sed muricus deroso flore carminis poema non canerem, sed garrirem, quo residentes auditores inter acernea pocula salute(m) bibentes insana Baccho iudice debaccharent];

ein beim anruf des schenkdieners verwantes skapja, vocativ eines nach art von fiskja, aurtja, haurnja, skattja etc. zu skap dolium oder haustrum gebildeten und semantisch altsächsischem scaprvard (in scenkion endi scapuuardos Hēl. 2033) zu vergleichenden nomens;

matjam jāh drigkam als der anfang oder refrain eines üblichen trinkliedes.

Bei der verwendung nun dieser worte als elemente eines lat. verses war folgendes verfahren gewissermassen geboten bez. möglich:

der dem latein abgehende diphthong von heils wurde als disyllabische vocalverbindung nachgesprochen;

das $j$ von scapja, matjam konnte der lat. norm gemäss durch silbischen laut ersetzt werden;

die endung des vocativischen fremdwortes scapja konnte nach dem muster von nauta, auriga etc. als kürze, aber auch nach dem muster der aus dem griechischen entlehnten vocative auf $-\bar{a}$ als länge gefasst werden;

das auslautende $h$ von $j \bar{a} h$ musste bei widergabe des wortes als dem lat. munde fremder sprachlaut vernachlässigt werden.

So aber entstand ein tadelloser hexameter (spondiacus mit dactylus im vierten fusse):

Inter he $\mid i l s$ goti $\mid$ cum scap $i \mid \bar{a}$ mati $\mid$ am jō $\mid$ drincam der sich nahezu unverderbt in unserer überlieferung vorfindet: nach abrechnung der von einem modernisierenden copisten eingeführten eils und matzia (mit tzi als die assibilierung bezeichnender schreibung) sind nur zwei correcturen, eine änderung von $-a$ in $-\bar{a}(=-a m)$ und von -an in -am, erforderlich. $\left.{ }^{1}\right)$

$\mathrm{Zu}$ beachten ist ferner, dass, mit rücksicht einerseits auf den diphthong und das $-s$ von heils, andrerseits auf die (nach Wredes Sprache der Ostgoten 165. 176) dem oștgot. des 6. jh.'s

1) Die in den (von Riese mit $\alpha$ bezeichneten) Schedae Divionenses stehende lesart drincam kann nicht als aus dem archetypus stammend gelten, weil diese Schedae eben ein apographon sind der (drincan bietenden) Salmasius'schen hs. 
zukommenden $\bar{e}$ (aus ai) und $s$-losen nominative, unser epigramm nicht als das erzeugnis eines in Italien ansässigen oder verweilenden dichters $\mathrm{zu}$ gelten hat (hierdurch wird auch dem von Möller für ja eingesetzten jad der boden entzogen).

In betreff der umstrittenen tendenz des lat. gedichtes

Inter heils etc.

Non audet quisquam dignos edicere versus.

Calliope madido trepidat se iungere Baccho,

Ne pedibus non stet ebria Musa suis. ${ }^{1}$ )

sei schliesslich noch bemerkt, dass hier weder mit Massmann und anderen an den stossseufzer eines dachstubenpoeten $\mathbf{z u}$ denken, den der Goten zecherlärm in seiner arbeit störte, noch mit Grabow u. a. (s. Zs. fda. anz.6, 374. 25, 104) an eine klage, dass unter dem gotischen regiment die poesie nicht gedeihen könne. Aus dem 3. und 4. vers 'die muse scheut die gesellschaft des sich betrinkenden Bacchus, dieweil sie fürchtet, infolge eines bei solchem zechgelage bekommenen rausches nicht mehr auf den füssen stehen bez. das richtige versmass innehalten zu können'2), ergibt sich, dass dem verfasser die situation eines dichters vorschwebte, der beim vortrag seiner geisteskinder im kreise zechender Goten sich der gefahr, sich selbst und seine kunst zu blamieren, aussetzte ${ }^{3}$ ) (wegen solcher vorträge vgl. die oben aus Fortunatus' Praefatio citierte stelle).

1) Luc. Mueller, Baehrens (s. dessen ausgabe der Poetae latini minores 4,363) und Riese fassen die beiden disticha wegen ihres verschiedenen metrums als jedes für sich ein carmen bildend. Doch dürfte der enge zusammenhang der in 3. 4 und 1. 2 ausgedrückten gedanken eine solche trennung nicht empfehlen, zumal eine variante von n. 160 der Anthologie die nämliche verbindung eines zwei hexameter enthaltenden und eines aus hexameter und pentameter bestehenden distichons aufweist.

2) Vgl. wegen stare pedibus die stelle in Greg. Turonensis' Historia Francorum 6, 46 (in den MG.): Conficitque (Chilpericus) duos libros, quorum versiculi debilis nullis pedibus subsistere possunt.

3) Auf grund des inhalts der vss. 3. 4 ist für das edicere von 2 eine in der jüngeren sprache nach dicere 'vortragen' dem compositum beigemessene bedeutung anzunehmen.

GRONINGEN.

W. VAN HELTEN. 